\title{
The NLRP3 Inflammasome and Diabetic Cardiomyopathy
}

\author{
Editorial to: "Rosuvastatin alleviates diabetic cardiomyopathy by inhibiting NLRP3 \\ inflammasome and MAPK pathways in a type 2 diabetes rat model" by Beibei Luo et al.
}

\author{
Lalit P. Singh
}

Published online: 14 November 2013

(C) Springer Science+Business Media New York 2013

Chronic hyperglycemia and dyslipidemia are primarily linked to various complications of diabetes, especially of type 2 . Diabetes mellitus, both type 1 and 2, accompanies cellular oxidative/nitrosative stress and insulin resistance/deficiency leading to generation of a chronic low-grade inflammatory environment within tissues. Recent studies have focused on the role of a protein platform called the NLRP3 inflammasome ((nucleotide-binding domain, leucine-rich repeats containing family, pyrin domain-containing-3 (NLRP3), its adaptor apoptotic speck protein containing a caspase recruitment domain (ASC) and pro-caspase-1)), which activates caspase-1 and interleukin-1beta (IL-1 $\beta$ ) [1-3]. Among the various inflammasomes, NLRP3 is widely activated in sterile inflammation (inflammation not involving infection) and is the most studied inflammasome. IL-1 $\beta$ is a highly inflammatory cytokine; therefore, its activity is regulated at multiple steps, including priming (expression), processing (activation) and exocytosis (release) [4]. Subsequently, pro-inflammatory IL- $1 \beta$ acts on its surface receptor, IL-1R, which may activate its own expression and other inflammatory cytokines generating reactive oxygen/nitrogen species (ROS/RNS) and cellular stress. IL-1 $\beta$ itself may propagate a feed forward loop maintaining chronic ROS/RNS stress, inflammation and premature cell death and disease progression of diabetes, including diabetic cardiomyopathy (DCM) (Fig. 1). As mentioned above, the NLRP3 inflammasome is considered as a sensor of cellular stress induced by various extrinsic (bacteria, virus,

L. P. Singh $(\bowtie)$

Department of Anatomy and Cell Biology, Wayne State University

School of Medicine, Detroit, MI 48201, USA

e-mail: plsingh@med.wayne.edu

L. P. Singh

Department of Ophthalmology, Wayne State University School of Medicine, Detroit, MI 48201, USA toxins, UV, and others) and intrinsic (metabolic imbalance, mitochondrial and endoplasmic reticulum stresses, organelle damage and oxidative stress) agents. Therefore, understanding the molecular mechanism(s) of the NLRP3 inflammasome assembly and activation is critical and currently constitutes an area of intense research in chronic metabolic diseases such as diabetes and obesity.

In this issue of Cardiovascular Drugs and Therapy, an article by Beibei et al. provides the first report that the NLRP3 inflammasome components are activated in a model of type 2 diabetes in rats and that NLRP3 knock down by microRNA in vivo ameliorates cardiac remodeling and dysfunction [5]. In addition, rosuvastatin (RSV), a statin inhibiting the HMG-CoA enzyme, blocks excessive expression of the thioredoxin interacting protein (TXNIP), a protein that is considered to be involved in cellular oxidative stress and the NLRP3 inflammasome assembly [1-3], and also showed that RSV reduces the level of NLRP3, caspase-1, and IL-1 $\beta$. RSV further suppresses activation of mitogen-activated protein kinases (MAPKs) such as ERK/p38 MAPK and JNK in this type 2 diabetes rat model. Nonetheless, a direct role of TXNIP in NLRP3 inflammasome assembly is yet to be fully understood [6] while TXNIP is known to cause pro-IL-1 $\beta$ and NLRP3 gene expression via nuclear factor NF- $\mathrm{kB}$ activation $[7,8]$. Moreover, the long-term use of RSV in diabetics may have deleterious side effects. The question then remains which of these molecules and signaling pathways generating oxidative stress and the low-grade inflammation (such as TXNIP, NLRP3, caspase-1, MAPK/NF-KB, IL-1 $\beta$ itself or its receptor IL-1R) are the best targets to treat chronic metabolic diseases. Whether anti-oxidant and anti-inflammatory drugs should be introduced as adjuvant therapies in type 1 and 2 diabetes is still debated. Several trials are also being conducted using neutralizing antibodies targeting both IL-1 $\beta$ and its receptor IL-1R in various chronic metabolic and inflammatory diseases [9-11]. 
Diabetes

Chronic Hyperglycemia/dyslipidemia

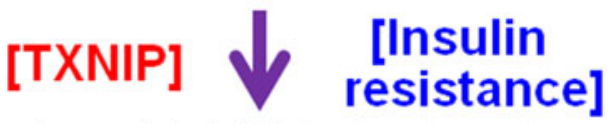

Mitochondrial ETC dysfunction

NADPH oxidase

Xanthene dehydrogenase/oxidase

Aberrant Iron metabolism

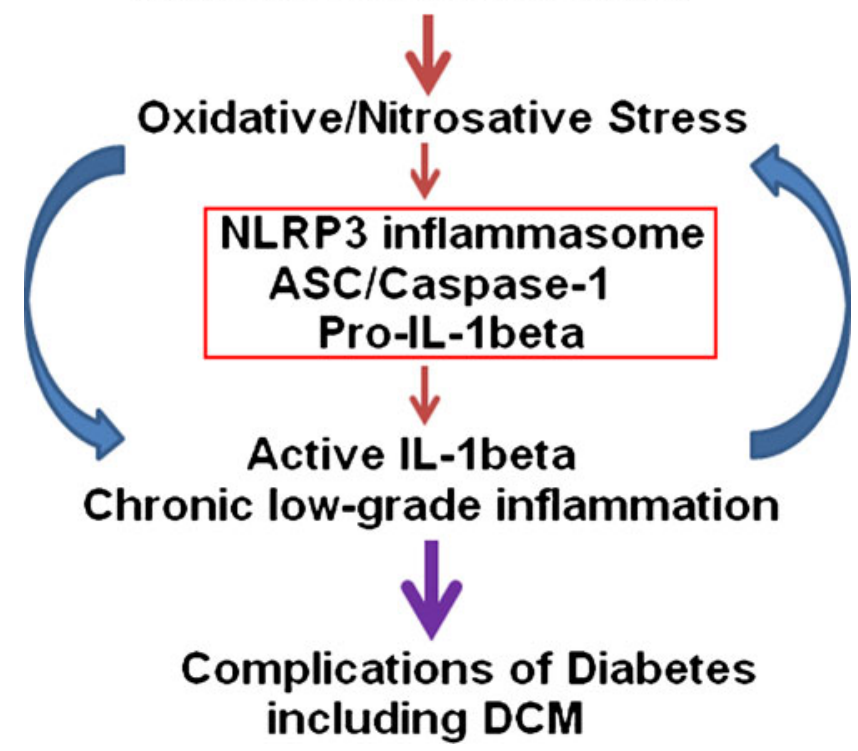

Fig. 1 Potential mechanism(s) for diabetes-induced NLRP3 inflammasome activation and cardiomyopathy. Diabetes increases TXNIP expression and insulin resistance causing cellular oxidative/ nitrosative stress through multiple sources leading to NLRP3 inflammasome assembly and pro-caspase-1 autocleavage/activation. Activated caspase- 1 in turn mediates pro-IL-1 $\beta$ processing and maturation. IL- $1 \beta$ is a potent inflammatory cytokine that can induce its own expression as well as other cytokines. Thus, a process of ROS/RNS stress and inflammation is produced in a self-sustained vicious cycle leading to cell injury and premature demise. Therapies aimed at blocking TXNIP, NLRP3 inflammasome and/or IL-1 $\beta$ may reduce or prevent progression of diabetic macro- and micro-vascular complications including DCM

In the future, in the opinion of this commentator, blocking early stress mediators such as TXNIP (as well as NLRP3 inflammasome components) that are significantly induced by hyperglycemia and dyslipidemia in diabetes and considered to play critical roles in ROS/ RNS stress, mitochondrial and ER stresses, inflammation and apoptosis will represent the best targets to prevent diabetes and slow down the progression of its complications including DCM [12-16].

\section{References}

1. Zhou R, Tardivel A, Thorens B, Choi I, Tschopp J. Thioredoxininteracting protein links oxidative stress to inflammasome activation. Nat Immunol. 2010;11(2):136-40.

2. Oslowski CM, Hara T, O’Sullivan-Murphy B, Kanekura K, Lu S, Hara M, et al. Thioredoxin-interacting protein mediates ER stressinduced $\beta$ cell death through initiation of the inflammasome. Cell Metab. 2012;16(2):265-73.

3. Lupfer C. Kanneganti TDUnsolved mysteries in NLR biology. Front Immunol. 2013;4:285.

4. Mankan AK, Dau T, Jenne D, Hornung V. The NLRP3/ASC/ Caspase-1 axis regulates IL-1 $\beta$ processing in neutrophils. Eur $\mathrm{J}$ Immunol. 2012;42(3):710-5.

5. Luo B, Li B, Wang W, Liu X, Liu X, Xia Y, Zhang C, Zhang Y, Zhang M, An F. Rosuvastatin alleviates diabetic cardiomyopathy by inhibiting NLRP3 inflammasome and MAPK pathways in a type 2 diabetes rat model. Cardiovasc Drugs Ther 2014;28: this issue

6. McGettrick AF, O'Neill LA. NLRP3 and IL-1 $\beta$ in macrophages as critical regulators of metabolic diseases. Diabetes Obes Metab. 2013;15(Suppl 3):19-25.

7. Bauernfeind FG, Horvath G, Stutz A, Alnemri ES, MacDonald K, Speert D, et al. Cutting edge: NF-kappaB activating pattern recognition and cytokine receptors license NLRP3 inflammasome activation by regulating NLRP3 expression. J Immunol. 2009;183(2):787-91.

8. Sbai O, Devi TS, Melone MA, Feron F, Khrestchatisky M, Singh LP, et al. RAGE-TXNIP axis is required for S100B-promoted Schwann cell migration, fibronectin expression and cytokine secretion. J Cell Sci. 2010;123(Pt 24):4332-9.

9. Moran A, Bundy B, Becker DJ, DiMeglio LA, Gitelman SE, et al. Interleukin-1 antagonism in type 1 diabetes of recent onset: two multicentre, randomised, double-blind, placebo-controlled trials. Lancet. 2013;381(9881):1905-15.

10. Hensen J, Howard CP, Walter V, Thuren T. Impact of interleukin-1 $\beta$ antibody (canakinumab) on glycaemic indicators in patients with type 2 diabetes mellitus: Results of secondary endpoints from a randomized, placebo-controlled trial. Diabetes Metab. 2013;S12623636(13)00130-4.

11. Dinarello CA, Simon A, van der Meer JW. Treating inflammation by blocking interleukin-1 in a broad spectrum of diseases. Nat Rev Drug Discov. 2012;11(8):633-52.

12. Xu G, Chen J, Jing G, Shalev A. Thioredoxin-interacting protein regulates insulin transcription through microRNA-204. Nat Med. 2013;19(9):1141-6.

13. Chen J, Cha-Molstad H, Szabo A, Shalev A. Diabetes induces and calcium channel blockers prevent cardiac expression of proapoptotic thioredoxin-interacting protein. Am J Physiol Endocrinol Metab. 2009;296(5):E1133-9.

14. Perrone L, Devi TS, Hosoya KI, Terasaki T, Singh LP. Inhibition of TXNIP expression in vivo blocks early pathologies of diabetic retinopathy. Cell Death Dis. 2010;1:e65.

15. Perrone L, Devi TS, Hosoya K, Terasaki T, Singh LP. Thioredoxin interacting protein (TXNIP) induces inflammation through chromatin modification in retinal capillary endothelial cells under diabetic conditions. J Cell Physiol. 2009;221(1):262-72.

16. Blouet C, Liu SM, Jo YH, Chua S, Schwartz GJ. TXNIP in Agrp neurons regulates adiposity, energy expenditure, and central leptin sensitivity. J Neurosci. 2012;32(29):9870-7. 